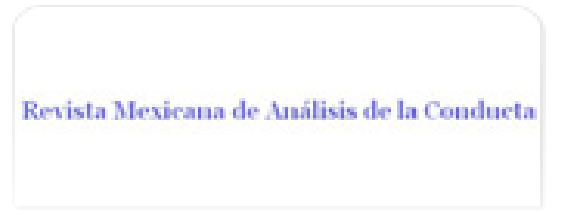

Revista Mexicana de Análisis de la Conducta ISSN: 0185-4534

editora@rmac-mx.org

Sociedad Mexicana de Análisis de la Conducta México

Guevara, Yolanda; Mares, Guadalupe; Rueda, Elena; Rivas, Olga; Sánchez, Beatriz; Rocha, Héctor Niveles de interacción que se propician en alumnos de educación primaria durante la enseñanza de la materia español

Revista Mexicana de Análisis de la Conducta, vol. 31, núm. 1, junio, 2005, pp. 23-45

Sociedad Mexicana de Análisis de la Conducta

Guadalajara, México

Disponible en: http://www.redalyc.org/articulo.oa?id=59331102

Cómo citar el artículo

- Número completo

- Más información del artículo

- Página de la revista en redalyc.org

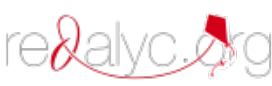

Sistema de Información Científica

Red de Revistas Científicas de América Latina, el Caribe, España y Portugal Proyecto académico sin fines de lucro, desarrollado bajo la iniciativa de acceso abierto 


\title{
NIVELES DE INTERACCIÓN QUE SE PROPICIAN EN ALUMNOS DE EDUCACIÓN PRIMARIA DURANTE LA ENSEÑANZA DE LA MATERIA ESPAÑOL
}

\author{
INTERACTION LEVELS FOSTERED DURING SPANISH LANGUAGE \\ CLASSES IN ELEMENTARY EDUCATION STUDENTS
}

\author{
YOLANDA GUEVARA, GUADALUPE MARES, ELENA RUEDA, \\ OLGA RIVAS, BEATRIZ SÁNCHEZ Y HÉCTOR ROCHA \\ FACULTAD DE ESTUDIOS SUPERIORES IZTACALA. \\ UNIVERSIDAD NACIONAL AUTÓNOMA DE MÉXICO
}

\begin{abstract}
RESUMEN
El objetivo fue analizar qué tipos de interacciones promueven las maestras de primaria durante las clases de la materia Español. Participaron nueve grupos de escuelas primarias públicas, tres de segundo, tres de cuarto y tres de sexto grado. En cada grupo se filmó una clase de aproximadamente una hora y se tomaron muestras de los productos académicos elaborados por los alumnos. Se registraron las actividades en cada momento de la clase y los niveles interactivos que se presentaban. Con base en la taxonomía de Ribes y López (1985) se ubicaron cinco niveles interactivos, de complejidad creciente, con diferentes probabilidades de generalización por parte de los alumnos. Los resultados muestran que la mayoría de las profesoras organizó actividades de lectura y copia -con lo que promueven interacciones en las cuales los niños se comportan como repetidores de información (nivel 1)-, alternando con preguntas que implican que los niños seleccionen partes de la lección (nivel 3). Se observó un descuido de actividades que implican habilidades instrumentales (nivel 2) e interacciones complejas de discusión y análisis (niveles 4 y 5). Estos resultados se comparan con los obtenidos en
\end{abstract}


un estudio previo que analiza las interacciones durante la enseñanza de las ciencias naturales y se discuten en términos de las posibilidades de generalización y aplicación de lo aprendido por los estudiantes.

Palabras clave: interacciones en el aula, aplicación y generalización del conocimiento, enseñanza del español, nivel funcional.

\begin{abstract}
The purpose of the present study was to analyze types of interaction fostered by elementary school teachers during Spanish language classes. Nine groups of public elementary schools participated (three from third grade, three from fourth grade and three from sixth grade). In each group, classes were videotaped for approximately one hour. Samples of academic products from the students were obtained. Activities and interactive levels were recorded in each moment of the class. Based on Ribes \& López taxonomy, 5 interactive levels characterized by increasing complexity and different generalization probabilities for the students were located. Results show that most teachers: a) organize reading and copying tasks which mainly foster interactions in which children merely repeat information (level 1); b) alternate questions which allow children to select sections of the lesson (level 3). Neglect for activities implying instrumental skills (level 2) and complex interaction involving discussion and analysis (levels 4 and 5 ) was observed. These results are compared with those from a previous study that analyzed interactions during natural science classes and are discussed in terms of students' possibilities of generalizing and applying what they have learned.

Key words: Teacher-students interactions, Elementary education, Teaching.
\end{abstract}

Desde una perspectiva del Análisis de la Conducta, y desde los años sesenta, se han realizado estudios para investigar los efectos que tiene el comportamiento del profesor sobre la conducta de los alumnos en el aula, tanto en lo relativo al aprendizaje como en aspectos de disciplina. Después de una revisión exhaustiva de la literatura conductual de tres décadas, Martens, Halperin, Rummel y Kilpatrick (1990) prueban que se cuenta con un extenso cuerpo de estudios que demuestra los efectos de la atención del maestro -incluyendo el uso de instrucciones, instigaciones, modelamiento, retroalimentación, corrección de errores y reconocimiento verbal- sobre la conducta de sus alumnos -en aspectos como frecuencia y duración de conductas problema, tiempo dedicado a la tarea y nivel de aprovechamiento-. El marco psicológico conductual ha enfatizado la importancia de las acciones pedagógicas del maestro para lograr un buen clima social en el aula y un buen aprovechamiento de los alumnos (Howell, Fox y Morehead, 1993; Orlich, Harder, 
Callahan, Kauchak y Gibson, 1994; Salvia y Hughes, 1990; Shea y Bauer, 1999; Shuell, 1996; Swanson, 1993; Taylor y Nolen, 1996; Wallace, Larsen y Elksnin, 1992).

En México, también se han llevado a cabo estudios encaminados a analizar las características de los salones de clase, las prácticas didácticas de profesores y los materiales de enseñanza utilizados en las escuelas primarias públicas (Alatorre, De Bengoechea, López, Mendiola y Sáiz, 1999; Guevara y Macotela, 2005; Guevara, Rivas, Rueda y Macotela, 1999; López y Rodríguez, 2003; Macotela y Jiménez, 1995; Schiefelbein y Farrés, UNESCO, 1991). En general, estos reportes indican deficiencias del sistema educativo mexicano en los tres aspectos señalados.

En lo relativo a la enseñanza del español a nivel de educación básica, los estudios realizados son escasos y de metodología diversa (Aiello y Olguín, 1998; Borzone y Rosemberg, 1994; Buxarrais, 1989; Castillo, Leos y Loza, 1999; Galeana, 1997; Molina, 1997; Thorne y Nakano, 2001); la mayoría de ellos tiene un enfoque psicopedagógico y se ha orientado principalmente a investigar el impacto de los estilos del profesor y sus prácticas didácticas sobre el comportamiento de los alumnos en clase, o bien sobre sus resultados en pruebas de ejecución académica.

A pesar del énfasis que se ha puesto en estudiar las prácticas didácticas y los estilos interactivos del profesor con sus alumnos, las investigaciones en el campo no han sido dirigidas a explorar el tipo de interacciones que se propician en los estudiantes de educación básica con respecto a los contenidos educativos de la clase.

Para ejemplificar distintos tipos de interacción que un profesor puede promover en clase, se señalará el caso de la lectura: un profesor puede leer un texto de corrido y los alumnos se concretan a escucharlo; otro, organiza una serie de actividades que demandan que los estudiantes sigan con la vista el texto que alguien lee y cambia constantemente al lector; uno más, puede alternar la lectura de párrafos con preguntas que lleven a los alumnos a vincular partes de los textos con experiencias vividas por ellos y relacionadas al contenido de la lección, a buscar de manera conciente e intencional el significado de algunas palabras, así como a vincular el párrafo recién leído con el párrafo anterior; otro profesor puede intercalar preguntas que orienten la atención hacia alguna definición y la elaboración de ejemplos distintos, etcétera. Estas actividades y tareas que el profesor organiza conllevan distintas maneras de entrar en contacto con los materiales impresos, de tal forma que los alumnos aprenden cosas distintas; para algunos, leer es sólo seguir con la vista, para otros leer es frenar la lectura, recordar aspectos que puedan vincularse con ella, buscar palabras desconocidas, encontrar nuevos ejemplos, de tal manera que la lectura queda integrada a un grupo amplio de experiencias vinculadas con la temática abordada. 
Distinguir los tipos de contacto que se propician en los salones de clase con los contenidos educativos, resulta relevante porque existe evidencia experimental que demuestra que el nivel de interacción que un alumno establece con los objetos de conocimiento (contenido de la clase) repercute en la posibilidad de generalizar las habilidades y destrezas ejercitadas (Mares, 1988; Mares, Guevara y Rueda, 1996; Mares, Ribes y Rueda, 1993; Mares, Rivas y Bazán, 2002; Mares, Rueda y Luna, 1990; Mares, Rueda, Plancarte y Guevara, 1997).

La línea de investigación encaminada a explorar los aspectos mencionados se enmarca en la perspectiva de campo interconductual (Kantor 1924/1969; Kantor, 1959/1978; Kantor y Smith, 1977) y toma como base la taxonomía de la conducta propuesta por Ribes y López (1985) para diferenciar los tipos de interacción. Dicha taxonomía propone cinco niveles de estructuración del comportamiento, de complejidad creciente, que se distinguen entre sí por el nivel de desligamiento funcional del organismo respecto a las propiedades de los eventos concretos y de la situación inmediata.

Las investigaciones de Mares y colaboradores han demostrado que: 1) cuando los niños son instruidos de manera exclusivamente verbal (por ejemplo, copiar y repetir información), se dificulta que entren en contacto con los aspectos referidos, lo cual a su vez disminuye la probabilidad de que el niño aplique dicho conocimiento en temas diferentes o en situaciones que le requieren habilidades distintas a la repetición, y 2) cuando los alumnos entran en contacto con los fenómenos en estudio, a través de actividades que les permitan elaborar relaciones entre eventos, son capaces de aplicar y generalizar lo aprendido a otras situaciones.

Con las mismas bases teórico-metodológicas, se diseñaron categorías para analizar las interacciones de madres e hijos en el hogar, con la finalidad de conocer el impacto de distintos tipos de interacción sobre habilidades lingüísticas de niños en desarrollo (Cortés y Delgado, 2002; Galicia, 1994; Guerrero, 1998; Guevara y Mares, 1994). Estas investigaciones mostraron que existe una relación entre los niveles interactivos que la madre promueve, a través de las actividades en las que se involucra con el niño, y los niveles de aptitud funcional que los niños desarrollan. En el estudio realizado por Guevara, Mares, Sánchez y Robles (1997) se lograron niveles más complejos de interacción en los niños, a través de un programa de intervención para modificar los niveles de interacción que las madres promueven.

Después de probar la utilidad de analizar las interacciones en términos de los niveles funcionales que las madres promueven, se desarrollaron categorías para estudiar las interacciones maestro-alumnos en el aula, tal como éstas ocurren durante la enseñanza de la lecto-escritura, en el primer grado de primaria en escuelas públicas (Mares, Bazán y Farfán, 1995; Mares, Reyes y Garrido, 2002). En estos estudios también se encontró una estrecha re- 
lación entre los niveles interactivos promovidos por las maestras y los grados de dominio alcanzados por los alumnos en tareas de lecto-escritura.

La taxonomía de Ribes y López, al aplicarse en el ámbito de la educación básica, considera que el nivel más simple de comportamiento (contextual) se presenta cuando los alumnos participan en actividades académicas que no requieren desligamiento de la situación presente, de tal manera que sólo se comportan como lectores, escuchas o repetidores de información. El segundo nivel (suplementario) implica cierto grado de desligamiento funcional, dado que el alumno debe realizar respuestas instrumentales ante instrucciones o modelos no presentes. El tercer nivel (selector) se da cuando el niño elige, de entre varias opciones, respuestas que se ajustan a un criterio específico que cambia de momento a momento, lo cual implica un mayor nivel de desligamiento funcional de situaciones presentes. El nivel cuatro (sustitutivo referencial) se presenta cuando los alumnos tienen interacciones que implican un desligamiento casi absoluto de la situación, al hacer referencia a eventos pasados o futuros cuando describen situaciones reales pero ausentes, este nivel implica el uso del lenguaje de manera convencional y ágil. El nivel más complejo se denomina sustitutivo no referencial y se distingue del anterior en que lo que se describe verbalmente no son objetos o situaciones reales, sino construcciones o modelos teóricos, y la convención lingüística que se utiliza implica el uso de terminología técnica o científica.

La integración de los hallazgos de las investigaciones precedentes permitió el desarrollo de una propuesta para analizar las actividades académicas durante la enseñanza de las Ciencias Naturales, en distintos grados de educación primaria, en términos de los niveles de interacción que las maestras promueven en los alumnos con los contenidos educativos (Mares y Guevara, 2004). Esta propuesta fue probada en nueve grupos de segundo, cuarto y sexto grados de escuelas públicas primarias (Mares, Guevara, Rueda, Rivas y Rocha, 2004). Los resultados de dicho estudio mostraron: 1) en los grupos de segundo y cuarto grados, la manera de llevar a cabo las clases de Ciencias Naturales promovió que los alumnos se comportaran principalmente como lectores, escuchas y repetidores de información (nivel contextual), y 2) las interacciones selectoras y las sustitutivo referenciales alcanzaron niveles altos sólo en un grupo de cuarto y dos de sexto grado. Esto implica un descuido de las actividades experimentales y analíticas que hubieran permitido a los alumnos tener una relación más cercana con los objetos de conocimiento.

Los datos arriba reportados constituyen un avance para conocer lo que sucede en los salones de clase, sus implicaciones para el aprendizaje y las posibilidades de generalización por parte de los alumnos. No obstante, se requiere realizar observaciones que, siguiendo la misma lógica, den cuenta de lo que ocurre en el aula durante la enseñanza de otras materias, porque exis- 
te evidencia que demuestra que un mismo educador promueve diferentes niveles interactivos dependiendo del contexto en el cual se desarrolla la interacción (Cortés y Delgado, 2002; Guevara, 1992; Guevara y Mares, 1994). En ese sentido, la materia de Ciencias Naturales representa un contexto distinto de la materia Español. Analizar las actividades e interacciones en las que los alumnos se involucran durante las clases de español es particularmente importante, porque en estos cursos se pretende sentar las bases sobre las cuales los alumnos manejarán el lenguaje oral y escrito, que se convertirán en herramientas para el aprendizaje de otras materias escolares.

La presente investigación tiene como objetivos:

1) Describir qué niveles de interacción promueven las maestras en los alumnos, a través de las actividades y tareas académicas que ellas organizan durante la enseñanza del español.

2) Describir cómo se modifican los niveles de interacción que las maestras promueven a lo largo de diferentes grados escolares (segundo, cuarto y sexto).

3) Analizar las implicaciones de los niveles de interacción organizadas por las maestras, con respecto a las posibilidades de generalización de las competencias promovidas en los alumnos durante las clases de español.

\section{MÉTODO}

La investigación consistió en un estudio prospectivo, de corte transversal, comparativo y observacional (Méndez, Namihira, Moreno y Sosa, 2001).

\section{Participantes}

Participaron nueve maestras con sus respectivos grupos de educación básica: tres de segundo, tres de cuarto y tres de sexto grado de primaria, pertenecientes a dos escuelas públicas, ubicadas en el municipio de Tlalnepantla, Estado de México. En lo que se refiere a los grupos de segundo, estuvieron presentes en la clase filmada 29 alumnos en el grupo 1, 35 en el 2 y 28 en el 3; la mayoría de ellos de 8 años de edad. En las clases de cuarto grado estuvieron presentes 29 alumnos en el grupo 4, 26 en el 5 y 27 en el 6; la mayor parte de ellos, de 10 años de edad. En los grupos de sexto grado estuvieron 16 alumnos en el grupo 7, 24 en el 8 y 28 en el 9; casi todos contaban con 12 años de edad.

Las escuelas y los grupos estudiados fueron aquellos cuyos directores y profesores mostraron disposición para participar en el estudio. Las escuelas están ubicada en una zona urbana con todos los servicios, y constituida principalmente por viviendas de interés social. 
Materiales

Cámara de videograbación, cassettes, hojas de registro, Plan de Estudios de la SEP, libros de avance programático, libros de texto y cuadernos de los alumnos.

Procedimiento de obtención de datos. Con el propósito de recolectar la información, se llevaron a cabo las actividades siguientes:

- Se filmó una clase por maestro sobre un tema de la materia Español; el tiempo de duración de la filmación correspondió a la duración de la clase.

- Se tomó una muestra de los productos académicos permanentes que los alumnos elaboraron durante la clase (copias de las páginas del libro de trabajo con los ejercicios elaborados por los niños, copias de su cuaderno, o similares) y se tomó registro de los materiales didácticos utilizados por el profesor.

\section{Registro de datos}

Se utilizó un formato de vaciado y análisis de datos de cada clase (Mares, Guevara, Rueda, Rivas y Rocha, 2004), que incluye: grupo, grado, escuela, profesora, número de alumnos presentes, fecha, duración, bloque del programa de estudios y objetivos curriculares de la sesión. En el mismo formato se incluyó un registro de: a) la frase que daba inicio al episodio interactivo, b) la descripción de la actividad, c) una descripción detallada de los segmentos de interacción ocurridos en el salón de clase, entre la maestra, los alumnos y los objetos de conocimiento, incluyendo una transcripción de los episodios de preguntas y respuestas, d) el material o recurso didáctico empleado por la maestra, e) la categoría ocurrida en cada segmento de interacción, y f) el tiempo que duró cada segmento.

Segmento o episodio interactivo: Está constituido por las interacciones que ocurren de manera sucesiva entre la maestra, los alumnos y los objetos de conocimiento, y que pertenecen a un mismo nivel funcional y a una misma categoría. En el Anexo 1 se describen las categorías de análisis utilizadas. En el caso de episodios constituidos por interacciones lingüísticas, la categoría se determina con base en la información que proporciona la maestra o el texto, las preguntas que la maestra formula y las respuestas que los niños dan; para el caso de la realización de ejercicios prácticos, además de lo anterior, se consideran los productos académicos que los alumnos realizan. El cambio de un segmento interactivo a otro (el corte del segmento) se registró cuando cambiaba la interacción. Los registros fueron realizados por observadores entrenados, pasantes de la carrera de Licenciatura en Psicología de la Facultad de Estudios Superiores, Iztacala.

Confiabilidad. Dos observadores analizaron cada videograbación, decidieron conjuntamente sobre la ubicación de cada segmento conductual en 
una categoría específica y llenaron el formato correspondiente, con todos los elementos presentes en la clase. Los videos y sus formatos fueron, además, revisados conjuntamente por las investigadoras a cargo.

Análisis de datos

Uno de los objetivos de la presente investigación fue explorar qué niveles de interacción propician las actividades que las maestras organizan durante la enseñanza del Español. Para lograr esto, se obtuvo el porcentaje de tiempo de la clase en que los alumnos mostraron cada nivel funcional ante las actividades que la maestra estructuraba, para cada grupo estudiado; con esto se obtuvo el patrón de interacciones por grupo, que permitió ver las categorías más frecuentes en cada clase.

Posteriormente, se compararon los patrones de interacción de los grupos, para analizar si pueden observarse cambios por grado escolar.

\section{Resultados}

La Figura 1 muestra los niveles de interacción promovidos por las maestras, a través de las actividades organizadas para las clases de español. La figura indica el porcentaje de tiempo de la clase dedicado a cada nivel. El anexo 2 contiene una descripción de las actividades por grupo.

Niveles de interacción promovidos en segundo grado

En dos de los grupos de segundo grado las actividades más frecuentes correspondieron al nivel contextual (grupos 2 y 3 ), mientras que en el grupo 1 , esta categoría, a pesar de no ser la más frecuente, se presentó durante el $20 \%$ del tiempo de la clase. En los grupos donde predominó el nivel contextual, las maestras leían un cuento sin interrupción, mientras los niños escuchaban y seguían la lectura. En el grupo 1 la maestra, en lugar de leer, narró el cuento, intercalando preguntas a lo largo de la narración. La posibilidad de participación de los alumnos del grupo 1, durante la exposición del cuento, marcó la diferencia en los porcentajes de tiempo dedicados a interacciones de nivel contextual. Además, en los tres grupos se pudo ubicar otra actividad que promovió interacciones contextuales, cuando los alumnos copiaron en sus cuadernos lo escrito por la maestra en el pizarrón.

En ninguno de los grupos de segundo grado se organizaron actividades que promovieran interacciones suplementarias de los niños con los contenidos educativos.

Sólo en el grupo 1 predominaron las actividades que favorecieron el nivel selector, mientras que en los grupos 2 y 3 estas actividades ocuparon alrededor del $20 \%$ de la clase. Este nivel se presentó cuando los alumnos contestaron preguntas que concretaban los conceptos definidos en la clase, atendiendo a criterios condicionales, o bien, cuando realizaron ejercicios en 


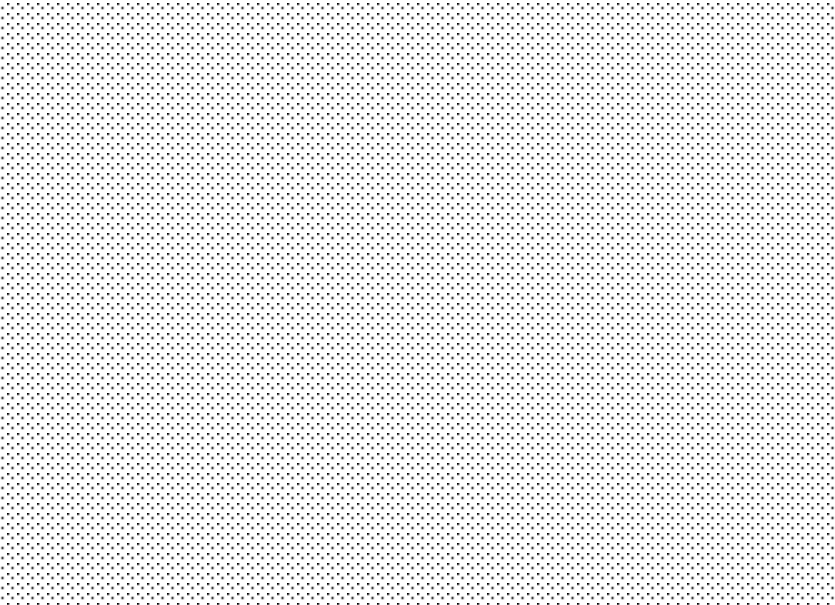

Grupos
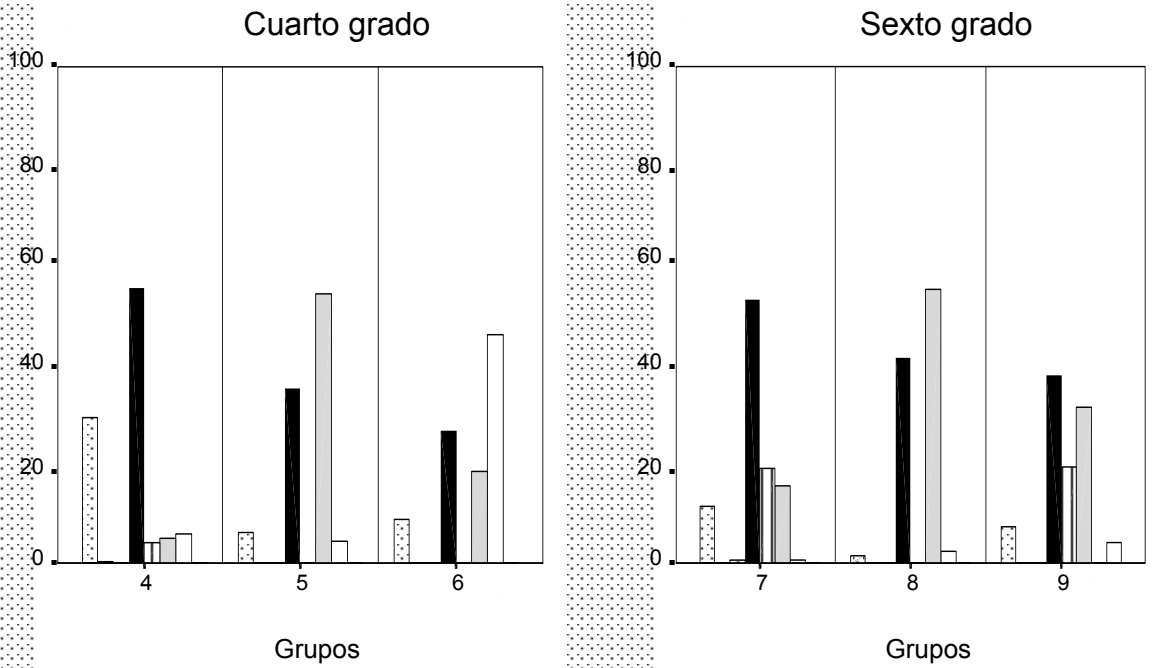

Figura 1: Muestra el porcentaje de tiempo de la clase que fue dedicado a actividades en cada nivel funcional, durante las clases de español, en segundo, cuarto y sexto grado. 
el libro de actividades, que implicaban la identificación de ejemplos de los conceptos abordados.

En el grupo 1 las actividades relacionadas con el nivel sustitutivo referencial prácticamente no ocurrieron, en el grupo 2 ocuparon menos del 10\% de la clase y en el grupo 3 la maestra invirtió cerca del $30 \%$ de la clase en la organización de este tipo de actividades. Este nivel de interacción se promovió cuando las maestras preguntaban a los alumnos de qué suponían que se trataba el cuento mientras veían una serie de dibujos que lo ilustraban. También se presentó este tipo de interacciones a través de la formulación de preguntas cuya respuesta debía deducirse del contenido del cuento.

Las actividades relacionadas con interacciones sustitutivas no referenciales estuvieron ausentes.

Niveles de interacción promovidos en cuarto grado

La maestra del grupo 4 fue la única, de todas las participantes en el estudio, que ocupó un alto porcentaje del tiempo de clase $(29 \%)$ en actividades de preparación.

En lo relativo al tiempo dedicado a actividades que promovieron interacciones a nivel contextual, la maestra del grupo 4 utilizó un $55 \%$ de la clase; durante ese tiempo leyó en voz alta un cuento que los alumnos siguieron en silencio y después pidió que algunos niños leyeran el mismo texto en voz alta y alternadamente. En el grupo 5, el tiempo de clase empleado en interacciones contextuales fue del $35 \%$, durante el cual la lectura de un cuento se alternó con explicaciones de la maestra. En el grupo 6, en lugar de leer, la profesora narró un cuento y explicó las partes de éste, a lo que dedicó el $27 \%$ del total de la clase.

Al igual que en los grupos de segundo grado, en los de cuarto las actividades suplementarias estuvieron prácticamente ausentes.

El nivel selector fue promovido en porcentajes muy bajos en el grupo 4. En cambio en el grupo 5 éste fue el nivel de interacción más promovido; casi el $60 \%$ de la clase fue empleado en que los niños, por parejas, resolvieran ejercicios seleccionando del texto las respuestas pertinentes, después de ello se realizó un ejercicio para corregir en grupo los errores, con ayuda de la maestra. Una actividad similar organizó la profesora del grupo 6, pero dedicándole menos tiempo de la clase, alrededor del $20 \%$.

Es lo relativo al nivel sustitutivo referencial donde se observaron mayores diferencias entre las clases de cuarto grado. Mientras que en los grupos 4 y 5 tales actividades estuvieron en niveles cercanos a cero, en el grupo 6 fueron las que predominaron; casi el $50 \%$ de esta clase se dedicó a que los alumnos respondieran en grupo preguntas relativas al cuento narrado y a que inventaran un cuento, escribiéndolo y señalando las partes que lo constituyen. 
Las interacciones sustitutivas no referenciales estuvieron ausentes en los tres grupos de cuarto grado.

\section{Niveles de interacción promovidos en sexto grado}

En los tres grupos de sexto se observaron porcentajes altos de interacciones contextuales (55, 40 y 40, respectivamente). En el grupo 7 fue éste el nivel de interacción predominante, la maestra organizó a los alumnos para que leyeran en voz alta una leyenda, ella resumió y expuso para clarificar el tema y definir palabras desconocidas para los alumnos; la mayor parte del tiempo, ellos escucharon o repitieron la información expuesta. En el grupo 8 la única diferencia fue que se abordó el tema de los derechos humanos y en el grupo 9 se leyó un cuento.

De todos los participantes en el estudio, los grupos 7 y 9 fueron los únicos que mostraron interacciones suplementarias. Este nivel se presentó cuando las maestras organizaron actividades que implicaban subrayar palabras con $g$ y $j$, encerrar en círculos los verbos y completar un ejercicio para transformar al infinitivo verbos conjugados. En estos dos grupos se empleó aproximadamente el $20 \%$ del tiempo de clase en dichas actividades.

Las interacciones selectoras se presentaron en los tres grupos de sexto grado, aunque con variaciones en sus porcentajes. El grupo 7 empleó casi el $20 \%$ del tiempo en contestar preguntas del tipo quién, dónde, qué, cuándo, que implicaban seleccionar las partes de la leyenda que habían leído previamente; el grupo 8 ocupó cerca del $60 \%$ de la clase en ejemplificar los derechos humanos, y el grupo 9 se involucró el $30 \%$ del tiempo en actividades selectoras que incluyeron buscar términos en el diccionario, definir cuándo se usa el guión en un texto y ubicar reglas ortográficas.

El nivel sustitutivo referencial se observó en el grupo 8 y el sustitutivo no referencial en el grupo 9, pero en ambos casos los porcentajes son muy bajos.

Como se desprende de los resultados descritos, no es posible identificar un patrón de interacciones característico de segundo, cuarto o sexto grados, ni pueden distinguirse cambios atribuibles al grado escolar, en ninguna de las categorías de observación. Esto implica que, durante la enseñanza de la materia Español, los recursos didácticos y la forma de estructurar las clases no varían de grado a grado. En la mayoría de los grupos se utilizó la lectura de un cuento como recurso didáctico y el tiempo dedicado a actividades de cada nivel funcional no parece ser influido por el grado escolar que se imparte.

\section{Discusión}

No se descarta la posibilidad de que, eventualmente, las maestras partici- 
pantes estructuren otro tipo de actividades y promuevan interacciones en el grupo con patrones diferentes a los aquí encontrados, nuestro análisis se centra en los hallazgos de las investigaciones aquí referidas y en particular los del presente estudio. Durante las clases filmadas, las maestras participantes organizaron principalmente actividades que promovieron interacciones de naturaleza contextual y selectora. La forma como estructuraron sus clases implicó frecuentemente alternar la lectura o exposición de una parte de la lección con un serie de preguntas dirigidas a que los alumnos eligieran términos, conceptos o ejemplos para contestar preguntas relacionadas con la lección, atendiendo criterios condicionales -particulares al concepto-. Es decir, alternar actividades que promueven interacciones contextuales con aquéllas que promueven un nivel selector.

Sólo en un grupo de cuarto grado predominaron las actividades que promueven interacciones sustitutivas referenciales y en otro grupo de segundo éstas correspondieron al $20 \%$ del tiempo de la clase. En siete grupos dicha categoría no se presentó de manera significativa. Es decir, la mayoría de los grupos estudiados no se involucró en actividades tales como: referir experiencias vinculadas con los temas tratados, hacer resúmenes con sus propias palabras de lo revisado o leído, deducir los contenidos a través de títulos, subtítulos e imágenes, elaborar distintos tipos de texto ajustándose a formatos definidos -cartas, telegramas, invitaciones o cuentos-.

Las actividades que permiten promover interacciones sustitutivas no referenciales estuvieron prácticamente ausentes. Puede decirse que los niños no se involucraron en discusiones o tareas que implican emitir juicios argumentados, generar reglas de convivencia en grupo o elaborar escritos utilizando lenguaje literario o técnico.

La forma en que las profesoras de este estudio estructuraron las clases de Español propicia que los alumnos empleen los conceptos propios de la materia de manera generalizada dentro de la misma situación. No obstante, este tipo de enseñanza no promueve la generalización hacia otras situaciones que pueden tener un nivel de exigencia mayor. Por ejemplo, cuando la maestra instruye a los niños en el análisis sintáctico de oraciones, posibilita que los niños analicen después oraciones diferentes (generalización intrasituacional), pero no promueve que los niños empleen dicha capacidad analítica para producir y corregir sus propios escritos (generalización extrasituacional). Para que estas habilidades de redacción puedan desarrollarse y generarse se requiere que la maestra involucre a los niños en actividades que impliquen la utilización de las competencias de análisis sintáctico en la elaboración de escritos propios, esto es, organizar en clase actividades que promuevan interacciones sustitutivas referenciales. Con este tipo de actividades se aumentaría la probabilidad de lograr el uso generalizado de las competencias analíticas en diferentes situaciones. 
Al comparar los hallazgos del presente estudio, correspondientes a la enseñanza del Español, con los reportados por Mares y cols. (2004) respecto a la enseñanza de las Ciencias Naturales, pueden señalarse algunos aspectos de interés:

En segundo y cuarto grados, las clases de Ciencias Naturales se organizaron promoviendo en los alumnos interacciones casi exclusivamente contextuales, donde los niños participaron escuchando la exposición y lectura de los temas, copiando ejercicios del pizarrón o repitiendo información; mientras que en las clases de Español, aun cuando las actividades contextuales se presentaron en porcentajes altos, la mayoría de las maestras organizó actividades que intercalaban la lectura y exposición de los temas con preguntas que promovieron interacciones selectoras.

En sexto grado, las clases de ciencias naturales, al igual que las de Español, fueron organizadas promoviendo niveles contextuales, pero intercalando actividades que implican relacionar términos técnicos, elaborar ejemplos para concretar conceptos y buscar respuestas a preguntas concretas, seleccionándolas de un texto o una exposición. Es decir, que las diferencias encontradas en los grados anteriores entre las clases de Ciencias Naturales y Español, disminuyen en el sexto grado.

Las diferencias antes descritas, sin ser concluyentes, pueden ser indicadores de que a las profesoras de educación primaria les puede resultar más difícil impartir cursos de Ciencias Naturales que de Español, particularmente en los primeros grados escolares. Esto, a su vez, puede relacionarse con los hallazgos de Paz (1999, 2000a, 2000b, 2001a y 2001b), quien reporta que los maestros suelen tener problemas para estructurar sus clases de ciencias naturales por las características del material de estudio.

De cualquier manera, independientemente de la materia, es evidente que en las clases analizadas la mayoría de las maestras no logran estructurar sus actividades de tal manera que aseguren que los alumnos vayan más allá de la repetición de los contenidos revisados.

El lenguaje -oral o escrito- tiene como principal característica la de poner al lector o escucha en contacto con objetos, personas o eventos no presentes en tiempo y espacio, a ello se le denomina nivel referencial del lenguaje. Como ya fue mencionado en la introducción de este escrito, los hallazgos de investigaciones previas indican que cuando los niños son instruidos de manera exclusivamente verbal (copia y repetición), sin entrar en contacto con los fenómenos en estudio, tienen problemas para generalizar o aplicar el conocimiento.

Como señala Ribes (1990), la inteligencia no es un evento mental oculto, sino que es una competencia como práctica, un saber cómo. Entonces, la enseñanza de las materias no puede concretarse a la repetición de características y contenidos, o a solicitar que los alumnos respondan una serie de 
preguntas. Tales recursos didácticos ayudan a los alumnos a lograr un primer contacto con los contenidos temáticos, y en el mejor de los casos, a manejar un discurso sobre los temas de la clase; sin embargo, no favorecen el desarrollo de competencias que implican el dominio y uso correcto y generalizado de los conceptos.

En el caso de la enseñanza de la materia Español, no basta con que los niños escuchen o lean un cuento y contesten preguntas sobre éste o sobre las reglas gramaticales que en él se utilizan. Es necesario llevar a cabo una serie de acciones que favorezcan diferentes niveles de interacción del alumno con los contenidos educativos, algunas de tales acciones son:

1. Que, previamente a la lección, el profesor solicite a sus alumnos la lectura de títulos y subtítulos. Desde hace varias décadas, autores como Rossi (1988) y Slater, Graves y Piché (1985) demostraron que a través del uso de subtítulos se mejoran los niveles de comprensión y recuerdo que los alumnos muestran sobre los aspectos referidos en el texto. Más tarde, y con estudios realizados desde una perspectiva interconductual, Rueda, Mares y Vallejo (1990) aportaron evidencias, con lectores iniciales, de que la inclusión de subtítulos y dibujos ilustrativos de cada tópico referido pueden ser suficientes para lograr la repetición de las expresiones lingüísticas contenidas en el texto, pero no para generalizarlas hacia un tema diferente. Esto lleva a la necesidad de incluir otras estrategias en la tarea educativa.

2. Que antes o durante la exposición o lectura de un tema, el profesor elabore preguntas a los alumnos para que refieran sus experiencias directas con los contenidos de la lección, y que después de ésta, promuevan en sus estudiantes el establecimiento de relaciones entre objetos, eventos y personas, así como la emisión de juicios argumentados con base en las reglas o definiciones vistas en esa y otras clases. Todo esto es importante, especialmente con niños de los primeros grados escolares, porque se requiere asegurar que los alumnos interactúen con los aspectos referidos en la lección y los incorporen a su discurso referencial. Mares, Guevara y Rueda (1996) probaron que para que el niño generalice lo aprendido a través de la lectura, se requiere asegurar su interacción con los aspectos referidos en el texto, a través del empleo de recursos tales como: a) preguntas que orienten su atención hacia las relaciones descritas, b) dibujos que ejemplifiquen cada una de dichas relaciones, y c) dibujos en los que falta la parte que representa la relación descrita en el texto y que sólo pueden ser completados por el alumno a través de la lectura.

3. Que los alumnos realicen ejercicios hasta que logren utilizar las reglas y conceptos para estructurar correctamente sus propios discursos orales y escritos, lo que favorece el perfeccionamiento cuantitativo y cualitativo dentro de cada competencia y cada nivel funcional. 
En suma, se hace necesario que los profesores de educación básica organicen sus clases llevando a los alumnos hacia el desarrollo de diversas competencias, y a través de los niveles funcionales sucesivamente más complejos. Si los discursos didácticos, los textos y las actividades escolares no garantizan el paso de los alumnos por distintos niveles funcionales, así como el logro del nivel referencial en las competencias académicas, difícilmente podrán cumplir su función formativa.

\section{REFERENCIAS}

Aiello, M. y Olguín, B. (1998). Las estrategias de enseñanza y de aprendizaje en el aula. Ethos Educativo, diciembre, 18, 57-68.

Alatorre, S., De Bengoechea, N., López, L., Mendiola, E. y Sáiz, M. (1999). Análisis de los materiales oficiales para la enseñanza de las matemáticas en primaria. $V$ Congreso Nacional de Investigación Educativa. Aguascalientes, 30 y 31 de octubre, 1 y 2 de noviembre. México.

Borzone, A. M. y Rosemberg, C. R. (1994). El intercambio verbal en el aula: las intervenciones de los niños en relación con el estilo de interacción del maestro. Infancia y Aprendizaje, 67-68, 115-132.

Buxarrais, M. R. (1989). Análisis de la interacción profesor-alumno como catalizadora del proceso de aprendizaje. Revista de Educación, enero-abril, 288, 419-428.

Castillo, T., Leos, M. y Loza, L. (1999). El estilo comunicativo del maestro y la participación del alumno. Revista CIPS, enero-abril 1999, 25-32.

Cortés, A. y Delgado U. (2002). Análisis funcional del desarrollo del lenguaje. En Guadalupe Mares y Yolanda Guevara (coordinadoras), Psicología Interconductual. Avances en investigación básica. México: Universidad Nacional Autónoma de México.

Galeana, C. R. (1997). La infancia desertora. México: Fundación SNTE para la Cultura del Maestro Mexicano, A. C.

Galicia, X. (1994). El uso de la palabra y su relación con la adquisición del lenguaje. Tesis inédita de maestría en Psicología: Metodología de la Teoría e Investigación Conductual. Universidad Nacional Autónoma de México. Facultad de Estudios Superiores, Iztacala. México.

Guerrero, P. L. (1998). Interacciones funcionales madre-hijo en díadas con y sin riesgo ambiental. Tesis inédita de Licenciatura en Psicología. Universidad Nacional Autónoma de México. Facultad de Estudios Superiores, Iztacala. México.

Guevara, Y. (1992). Tesis inédita de Maestría en Psicología. Análisis funcional de las interacciones lingüísticas del niño con retardo en el desarrollo. Universidad Nacional Autónoma de México. Facultad de Estudios Superiores Iztacala. México.

— - Y. y Macotela, S. (2005). Escuela: del fracaso al éxito. Cómo lograrlo apoyándose en la psicología. México: Pax.

_ _, Y. y Mares, G. (1994). Análisis de las interacciones madre-hijo retardado: una 
perspectiva interconductual. Acta Comportamentalia, 2, 145-165.

_ _, Y., Mares, G. Sánchez, B. y Robles, S. (1997). Modificación de patrones interactivos madre-hijo retardado. Revista Latina de Pensamiento y Lenguaje, 5, 2, 105-137.

—_, Y., Rivas, O., Rueda, E. y Macotela, S. (1999). Análisis de las prácticas didácticas de maestros de primer grado: un estudio descriptivo. Revista Integración. Educación y Desarrollo Psicológico, 11, 78-95.

Howell, K., Fox, S. y Morehead, M (1993). Curriculum-based evaluation: teaching and decision making. California: Brooks/Cole Co.

Kantor, J. R. (1924/ 1969). Principles of Psychology. Granville, Ohio, The Principia Press.

—. (1959/1978). Psicología Interconductual. Un ejemplo de construcción científica sistemática. México: Editorial Trillas.

— _ \& Smith, N. W. (1977). The Science of Psychology. An interbehavioral survey. Chicago, The Principia Press, Inc.

López, G. y Rodríguez, M. (2003). La evaluación alternativa: oportunidades y desafíos para evaluar la lectura. Revista Mexicana de Investigación Educativa, 8, 17,6798.

Macotela, S. y Jiménez, E. (1995). Una perspectiva sistémica para la participación del psicólogo en problemas asociados al fracaso escolar. En G. Acle (Ed.) Educación especial. Evaluación, intervención e investigación. México: Universidad Nacional Autónoma de México.

Mares, G. (1988). Análisis experimental de la relación entre diferentes competencias lingüísticas. Tesis de Maestría en Análisis Experimental de la Conducta. Facultad de Psicología. México: Universidad Nacional Autónoma de México.

—_, Bazán, A. y Farfán E. (1995). Adecuación y evaluación de un programa interconductual para la enseñanza de la lengua escrita. Desarrollo Académico. Universidad Pedagógica Nacional, Año 3, 14-21.

—_. y Guevara, Y. (2004). Propuesta para analizar la práctica educativa durante la enseñanza de las Ciencias Naturales. En J. J. Irigoyen (Ed.) Análisis funcional del comportamiento. México: Universidad de Sonora.

_- Guevara, Y. y Rueda, E. (1996). Modificación de las referencias orales y escritas a través de un entrenamiento en lectura. Revista Interamericana de Psicología, 30, 2, 189-207.

—-, Guevara, Y., Rueda, E. Rivas, O. y Rocha, H. (2004). Análisis de las interacciones maestra-alumnos durante la enseñanza de las Ciencias Naturales en Educación Primaria. Revista Mexicana de Investigación Educativa, 9, julio-septiembre, 22, 721-745.

_- Reyes, A. y Garrido, A. (2002). Interactions between teacher-pupil and academic achievement. Sixth International Congress on Behaviorism and the Sciences of Behavior, United States of America.

_- Ribes, E. y Rueda, E. (1993). El nivel de funcionalidad en lectura y su efecto sobre la transferencia de lo leído. Revista Sonorense de Psicología, 7, 1, 32-43.

- Rivas. O. y Bazán, A. (2002). Configuración en el modo escrito de competencias ejercitadas en forma oral como efecto del nivel funcional de ejercicio del modo escrito. Revista Mexicana de Análisis de la Conducta, 28, 1, 173-201. 
—-, Rueda, E. y Luna, S. (1990). Grados de transferencia de los estilos lingüísticos en tareas referenciales. Revista Sonorense de Psicología, 4, 84-97.

—-, Rueda, E., Plancarte, P. y Guevara, Y. (1997). Conducta referencial no entrenada: el papel que juega el nivel funcional de entrenamiento en la generalización. Acta Comportamentalia, 5, 199- 219.

Martens, B., Halperin, S. Rummel, J. \& Kilpatrick, D. (1990). Matching theory applied to contingent teacher attention. Behavioral Assessment, 12, 139-155.

Méndez, I., Namihira, D., Moreno, L. y Sosa, C. (2001). El Protocolo de Investigación. México: Trillas.

Molina, S. (1997). El fracaso en el aprendizaje escolar. Málaga, España: Aljibe, Colección: Biblioteca de Educación.

Orlich, D., Harder, R., Callahan, R., Kauchak, D. \& Gibson, H. (1994). Teaching Strategies. A guide to better instruction. Massachusetts: D. C. Heath and Company.

Paz, R. (1999). Un diagnóstico del nivel de conocimientos básicos en biología del niño, al ingresar a primero de secundaria. Xictli, 36. http://www.unidad094.upn. $\mathrm{mx} /$ revista/xictli.htm

- - (2000-a). Algunas consideraciones sobre la enseñanza de la ciencia en la educación primaria y la necesidad de los docentes de acceder a una formación contínua efectiva. Xictli, 38. http://www.unidad094.upn.mx/revista/xictli.htm

— - (2000-b). ¿Qué saben los maestros sobre la biología en primaria? Xictli, 40. http://www.unidad094.upn.mx/revista/xictli.htm

—_. (2001-a). La enseñanza de la evolución en la educación primaria como una evidencia de los obstáculos a los que se enfrenta el niño para construir conceptos complejos. Xictli, 42. http://www.unidad094.upn.mx/revista/xictli.htm

- (2001-b). El análisis de la construcción conceptual como una herramienta para disciplinar las etapas del desarrollo del niño, un caso, el concepto de digestión. Xictli, 43, http://www.unidad094.upn.mx/revista/xictli.htm

Ribes, E. (1990). Aptitudes sustitutivas y la planeación del comportamiento inteligente en las instituciones educativas. En E. Ribes, Psicología general. México: Trillas.

- y López, F. (1985) Teoría de la Conducta. Un análisis de campo y paramétrico. México: Trillas.

Rossi, J. (1988). Text comprehension: macrostructure and frame. I/ European Meeting on the Experimental Analysis of Behavior. Lieja, julio 1988, Bélgica.

Salvia, J. \& Hughes, Ch. (1990). Currículo-based assessment. Testing what is taught. New York: MacMillan Publishing Co.

Schiefelbein, E. y Farrés, P. (1991). Evaluación formativa de libros de texto de educación primaria. UNESCO. Revista Latinoamericana de Estudios Educativos. XXI, 2, 63-87.

Shea, T. M. y Bauer, A. M. (1999). Educación especial. Un enfoque ecológico. México: McGraw Hill.

Shuell, T. (1996). The role of educational psychoogy in the preparation of teachers. Educational Psychologist, 3, 1, 5-14.

Slater, W., Graves, M. \& Piché, G. (1985). Effects of structural organizers on minthgrade students'comprehension and recall of four patterns of expository text. Reading Research Quarterly, 20, 189-202.

Swanson, H. (1993). Principles and procedures in strategy use. In L. J. Meltzer (Dir.) 
Strategy assessment and instruction for students with learning disabilities. From theory to practices. Austin, Texas: Pro-Ed, Inc.

Taylor, C. \& Nolen, S. (1996). A contextualized approach to teaching teachers about classroom-based assessment. Educational Psychologist, 3, 1, 77-88.

Thorne, C. y Nakano, T. (2001). El rol del maestro de primer grado en el aprendizaje de la lectura en Perú. En A. Bazán (Ed.) Enseñanza y evaluación de la lectura y escritura. Algunos aportes de la investigación en psicología. México: Instituto Tecnológico de Sonora-Consejo Nacional de Ciencia y Tecnología.

Wallace, G., Larsen, S. \& Elksnin, L. (1992). Educational Assessment of Learning Problems. Austin, Texas: PRO-ED.

\section{ANEXO 1}

Categorías de análisis:

0) Actividades no contempladas directamente en los objetivos educativos.

0a) Actividades de preparación de la clase o de cambio de actividad. Es el tiempo que se emplea durante la clase para que los niños saquen y guarden materiales, se organicen en equipos y para que la maestra organice los materiales de la actividad siguiente.

ob) Actividad sin supervisión por parte de la maestra. Tiempo que los niños permanecen en el salón de clases sin la presencia de la maestra o cuando la maestra se encuentra atendiendo a otra persona ajena al salón de clases.

0c) Actividad no pertinente. Es cuando la maestra organiza una actividad que no está contemplada en los objetivos educativos.

1) Actividades que propician interacciones contextuales.

1a) La maestra lee o expone un tema (con o sin imágenes), y los niños siguen la exposición o lectura en el texto.

1b) Después de la presentación de cierta información, la maestra solicita que los niños repitan lo que se acaba de decir o leer. Los niños realizan la actividad. La interacción se concentra en los productos lingüísticos.

1c) La maestra solicita que los niños realicen cualquier actividad práctica que implica copiar lo escrito en el pizarrón o en algún libro y los niños lo llevan a cabo.

2) Actividades que favorecen interacciones suplementarias. 
2a) La maestra da instrucciones a los alumnos para que realicen una tarea que implica completar un ejercicio (subrayar, encerrar en círculos o relacionar columnas) o bien, aplicar un procedimiento; los niños lo realizan sin tener un modelo presente.

3) Actividades que promueven interacciones selectoras.

3a) La maestra elabora preguntas que requieren que el niño elija la parte de un producto lingüístico o de una imagen, de manera condicional a la palabra-pregunta. El niño copia, repite o elige el segmento pertinente. Por ejemplo, ante un texto, la maestra elabora preguntas del tipo "quién", "cuándo", "dónde", "por qué", "qué" de tal manera que la selección de segmentos del texto se vuelva relativa al tipo de palabra-pregunta formulada. Otro ejemplo sería seleccionar de un texto palabras con diferentes funciones gramaticales.

3b) La maestra pide a los alumnos que elaboren ejemplos para concretar conceptos; los niños participan eligiendo los ejemplos pertinentes. La maestra les solicita que elaboren resúmenes y los alumnos eligen partes del texto.

3c) La maestra solicita que los niños expliquen, con base en reglas gramaticales o en definiciones, ciertas estructuras lingüísticas o equivalencias entre términos o expresiones. Por ejemplo, argumentar con base en la regla gramatical por qué peces se escribe con "c" si pez se escribe con " $z$ ", o bien, qué significa una palabra.

4) Actividades que propician interacciones sustitutivo referenciales.

4a) Antes o durante la exposición o lectura de un tema, la maestra elabora preguntas para que los niños refieran sus experiencias directas con los contenidos de la lección. Uno o varios niños lo hacen.

4b) Antes o durante la exposición o lectura de un tema, la maestra pide que los alumnos digan de qué suponen que trata la lección, uno o varios niños lo hacen. Por ejemplo, a través de una imagen o un título los alumnos deducen el contenido.

4c) La maestra pide a los alumnos que elaboren crónicas, descripciones, composiciones, ensayos, recetas, instructivos, cartas, telegramas, cuentos o producciones similares, a nivel oral o escrito. Uno o varios alumnos lo hacen.

5) Actividades que favorecen interacciones sustitutivo no referenciales.

5a) La maestra solicita que los niños emitan un juicio argumentando con base en reglas o definiciones, o bien, que elaboren reglas de relación entre ellos para producir cambios en el grupo; uno o varios niños participan en ello, argumentando sus ideas. 
5b) La maestra solicita que los alumnos elaboren ensayos, poemas o producciones similares a nivel oral o escrito. Los niños realizan la tarea utilizando lenguaje literario o terminología técnica.

\section{ANEXO 2}

\section{Actividades por grupo}

En el grupo 1 se abordó el tema "Antónimos". El objetivo del programa para esta lección es que los niños descubran que algunas palabras (sustantivos, adjetivos y verbos) pueden tener significados contrarios, permitiéndoles enriquecer su vocabulario. La maestra inició su clase contando u $\mathrm{n}$ cuento de un rey llamado Antónimo, en el cual resaltó palabras con significados opuestos; promovió que los alumnos dedujeran las palabras opuestas a través de preguntas e instigaciones y que definieran el concepto, anotando esto en el pizarrón para que los niños lo copiaran en sus cuadernos. Solicitó que escribieran ejemplos y que realizaran ejercicios en su libro, relacionados con cambiar una serie de palabras a sus respectivos antónimos. Al final, cantaron una canción ejercitando lo visto en clase.

En el grupo 2 el tópico a tratar fue "Semejanzas y diferencias". Los objetivos del programa para esta lección son: 1) activación de conocimientos previos, el niño deberá realizar predicciones acerca del contenido, a través de imágenes, y 2) que los niños describan la forma de ser de los personajes que más les hayan gustado del cuento, a través de adjetivos calificativos, identificando semejanzas y diferencias. Al inicio de la clase, la maestra mostró brevemente una lámina y después empleó algunos minutos en que los alumnos observaran las ilustraciones de la lección contenida en su libro de texto; pidió algunas descripciones y solicitó que los niños dijeran de qué suponían que trataba la historia. Después, la maestra leyó el cuento en voz alta sin interrupciones, mientras los alumnos seguían la lectura en silencio; al terminar de leer, realizó una serie de preguntas que implicaban repetir las características de los personajes, mientras ella exponía las semejanzas y diferencias. Por último, pidió a los alumnos que realizaran ejercicios en su libro de actividades.

El grupo 3 abordó "Lo real y lo fantástico" como tema. Los objetivos del programa para esta lección son: 1) activación de conocimientos previos, el niño deberá realizar predicciones acerca del contenido, 2) comprensión global, el niño aprenderá a identificar lo que es real y lo que es fantástico en un cuento. La estructura de esta clase fue muy similar a la del grupo 2, excepto porque en este grupo la lectura en voz alta se realizó alternando al lector, entre la maestra y diferentes alumnos. 
En el grupo 4 se utilizó la lectura "El nacimiento del sol y la luna". Los objetivos del programa para esta lección son: 1) que el alumno lea y comente el texto, narración de sucesos reales o ficticios; 2) identificación del sujeto en diferentes partes de la oración; 3) revisión y corrección de textos propios, y 4) participación en juegos que propicien el desarrollo de la lengua hablada y escrita. Al igual que las maestras de los grupos 2 y 3 , esta profesora inició la clase mostrando brevemente una lámina y pidiendo a los alumnos que observaran las ilustraciones de la lección contenida en su libro de texto, para que dijeran de qué suponían que trataba la historia, en lo que se invirtieron algunos minutos de la clase. Después, leyó el cuento en voz alta sin interrupciones y los alumnos siguieron la lectura en silencio; al terminar de leer el cuento, pidió que los alumnos volvieran a leerlo en voz alta, alternándolos para ello. Dio a los alumnos varios sobres con preguntas sobre el tema, y organizó un concurso entre equipos, relativo a contestar preguntas literales acerca del cuento. Por último, pidió a los alumnos que en una hoja escribieran un comentario sobre la lectura y lo ilustraran. Se dio por concluida la clase.

El grupo 5 leyó el texto "El ratón del supermercado y sus primos del campo". Los objetivos del programa para esta lección son: 1) lectura y comprensión de textos, 2) realizar comentarios sobre el tema, 3) realización de actividades en el libro, consultando preguntas en el texto, y 4) análisis de palabras cuyo significado depende de su escritura y separación correcta. Al inicio de la clase la maestra preguntó qué sabían los niños acerca de los ratones y dónde los habían visto. Pidió que leyeran varios párrafos de la lección, alternando los lectores, después realizó una serie de preguntas literales acerca de lo leído. El grupo llevó a cabo ejercicios en sus libros de actividades, relativos a palabras específicas vistas en la lección y la maestra corrigió los errores. Anotó oraciones en el pizarrón y explicó la diferencia que hay entre la forma de escribir las palabras, ya que su significado puede cambiar. Al concluir la exposición, cada niño realizó ejercicios en sus libros de actividades.

El grupo 6 se basó en una historia llamada "Los lentes de don Rufino". Los objetivos del programa para esta lección son: 1) lectura y comentarios de cuentos, 2) identificación del uso del sujeto tácito en oraciones y textos propios, 3) corrección individual y colectiva de textos, 4) narración y descripción oral de sucesos reales o ficticios, a partir de la lectura de un cuento, 5) creación de cuentos a partir de otros semejantes, y 6) modificación del final de algunos cuentos. La maestra inició la clase contando el cuento a los niños, con ayuda de dibujos en cartulinas pegadas en el pizarrón; a lo largo de la narración hizo varias pausas y realizó preguntas relacionadas con la historia. Al terminar la narración, la maestra hizo preguntas a los niños, del tipo “¿cómo se llamó el cuento?”, “¿dónde se desarrolló?”, etc. Después explicó las partes de un cuento, ejemplificando con el narrado; repartió hojas con un cuento diferente para que cada alumno lo leyera en silencio y contestara una 
serie de preguntas acerca del contenido y de las partes del cuento. Como última actividad, la maestra solicitó que cada niño escribiera un cuento y marcara las partes del mismo, en lo que se invirtió gran parte de la clase. La maestra revisó los escritos de los alumnos y proporcionó retroalimentación a varios de ellos.

El grupo 7 utilizó el texto "La leyenda del fuego". Los objetivos para esta lección se relacionan con: intercambio de ideas, las expresiones y su significado, investigación, las partes de una narración, escribir un cuento, antología de tradiciones mexicanas, ortografía (uso de la c y la q), acerca de la lengua (el pospretérito) y "para sentir la literatura". La clase inició con la lectura en voz alta, alternando los lectores; la maestra intercaló explicaciones y preguntas que implicaron respuestas literales de los niños. Después, explicó las partes de una narración y elaboró preguntas que implicaban seleccionar respuestas del texto leído. Durante toda la clase se alternaron explicaciones y preguntas. Los alumnos escribieron las respuestas en sus libros de actividades y la maestra revisó respuestas y corrigió ortografía.

El grupo 8 trató el tema de los "Derechos Humanos". Los objetivos de esta lección se relacionan con: interpretaciones del texto, intercambio de ideas, investigación, los formatos, documentos legales, ortografía (uso de los dos puntos), acerca de la lengua (las partes de la oración), organizar un concurso. La maestra pidió que los alumnos leyeran los derechos humanos, asignando un artículo por lector; después solicitó que dijeran el significado de ciertas palabras poco comunes, algunas de ellas se buscaron en el diccionario. La mayor parte de la clase trascurrió leyendo cada artículo y encontrando ejemplos que ilustraban éstos; frecuentemente la maestra expresaba sus opiniones, y ocasionalmente también los alumnos lo hacían. Posteriormente, los alumnos pasaron al pizarrón, alternadamente, a escribir cada uno de los derechos y obligaciones de los humanos, y a escribir los ejemplos de éstos. Para finalizar la clase, los alumnos copiaron en sus cuadernos lo escrito en el pizarrón.

El grupo 9 se basó en el texto "Gesto noble". Los objetivos del programa para esta lección se relacionan con: intercambio de ideas, las palabras y su significado, las partes del texto, investigación, interpretaciones del texto, escribir resúmenes, acerca de la lengua (campos semánticos) y ortografía (uso de la $g$ y la $j$ ). Se inició con la lectura en voz alta por parte de un alumno, cambiando al lector en cinco ocasiones, pero por problemas en la calidad de la lectura oral la maestra terminó leyendo casi todo el texto. Una alumna hizo un resumen de lo leído y se buscó en el diccionario el significado de algunas palabras. La maestra preguntó qué les había enseñado el cuento y algunos alumnos participaron. Después, cada estudiante realizó en su libro de actividades un ejercicio de localización de las partes de un texto y de ejemplos de guión largo, $g$ y $j$. En actividad grupal, la maestra pidió ejemplos de verbos 
en infinitivo y de la regla ortográfica en revisión, frecuentemente ella daba las respuestas y los alumnos las escribían en el pizarrón o las copiaban en su libro de actividades. Por último, los alumnos intercambiaron libros para calificarse entre sí y la maestra corroboraba las calificaciones. 